\title{
Reversible Cerebral Vasoconstriction Syndrome in a Patient with Takayasu's Arteritis
}

\author{
Yuto Uchida ${ }^{1}$, Noriyuki Matsukawa ${ }^{1}$, Takuya Oguri ${ }^{1}$, Keita Sakurai ${ }^{2}$, Toshiyasu Miura ${ }^{1}$, \\ Shiho Iwagaitsu ${ }^{3}$, Taio Naniwa ${ }^{3}$ and Kosei Ojika ${ }^{1}$
}

\begin{abstract}
We herein present the first reported case of reversible cerebral vasoconstriction syndrome (RCVS) in Takayasu's arteritis (TA), in a patient with severe thunderclap headache and generalized tonic-clonic seizure. Magnetic resonance imaging and angiography revealed posterior reversible encephalopathy syndrome, followed by severe vasoconstriction in multiple arteries. RCVS should be included in the differential diagnosis of thunderclap headache attacks with radiologic intracranial vascular abnormalities in connective tissue diseases, including TA.
\end{abstract}

Key words: reversible cerebral vasoconstriction syndrome, Takayasu's arteritis, intracranial vessel, magnetic resonance angiography, posterior reversible encephalopathy syndrome

(Intern Med 50: 1611-1614, 2011)

(DOI: 10.2169/internalmedicine.50.5185)

\section{Introduction}

Reversible cerebral vasoconstriction syndrome (RCVS) is characterized by prolonged but reversible vasoconstriction of the cerebral arteries, usually associated with acute-onset, severe "thunderclap headache", with or without additional neurologic signs and symptoms. The "string of beads" appearance of the cerebral arteries on angiographic images can resolve spontaneously in 1-3 months $(1,2)$. Previous reports have described the most common clinical feature to be a severe acute headache, like a "clap of thunder", similar to that of a ruptured aneurysm. However, cases described as RCVS could include a heterogeneous range of disorders. Furthermore, the underlying pathologic mechanism remains unknown (1).

Takayasu's arteritis (TA), which is common in Asia and Mexico, is a systematic inflammatory disease that affects the aorta and its main branches $(3,4)$. The inflammatory processes cause narrowing, occlusion, and/or dilation of arteries, resulting in a variety of symptoms. The involvement of the extracranial portions of the carotid and vertebral arteries induces cerebrovascular manifestations, e.g., vertigo, syncope, and convulsions, mainly caused by cerebral ischemia $(3,4)$. However, the involvement of intracranial arteries in patients with TA has rarely been reported (5). We herein report the presence of RCVS, and associated clinical and arteriographic involvement of the intracranial arteries, in a patient with TA. To our knowledge, this is the first case of RCVS described in a TA patient.

\section{Case Report}

A 15-year-old girl with TA presented to our emergency department complaining of acute-onset severe thunderclap headache with impaired consciousness, followed by generalized tonic-clonic seizure lasting a few minutes. She had been diagnosed with TA 3 months earlier, based on the American College of Rheumatology criteria. On that occasion, she had complained of low-grade fever, fatigue, and abdominal pain. Left radial arterial pulsations were diminished, and blood pressure in her left arm was reduced (right: 126/64 mmHg, left: 104/48 mmHg). Laboratory findings were as follows: microcytic anemia (hemoglobin: $8.1 \mathrm{~g} / \mathrm{dL}$ ), increased erythrocyte sedimentation rate $(130 \mathrm{~mm} / \mathrm{hr})$ and C-reactive protein $(8.03 \mathrm{mg} / \mathrm{dL})$. Contrast-enhanced com-

${ }^{1}$ Department of Neurology, Nagoya City University, Japan, ${ }^{2}$ Department of Radiology, Nagoya City University, Japan and ${ }^{3}$ Department of Medical Oncology and Immunology, Nagoya City University, Japan

Received for publication January 20, 2011; Accepted for publication April 4, 2011

Correspondence to Dr. Noriyuki Matsukawa, norim@med.nagoya-cu.ac.jp 


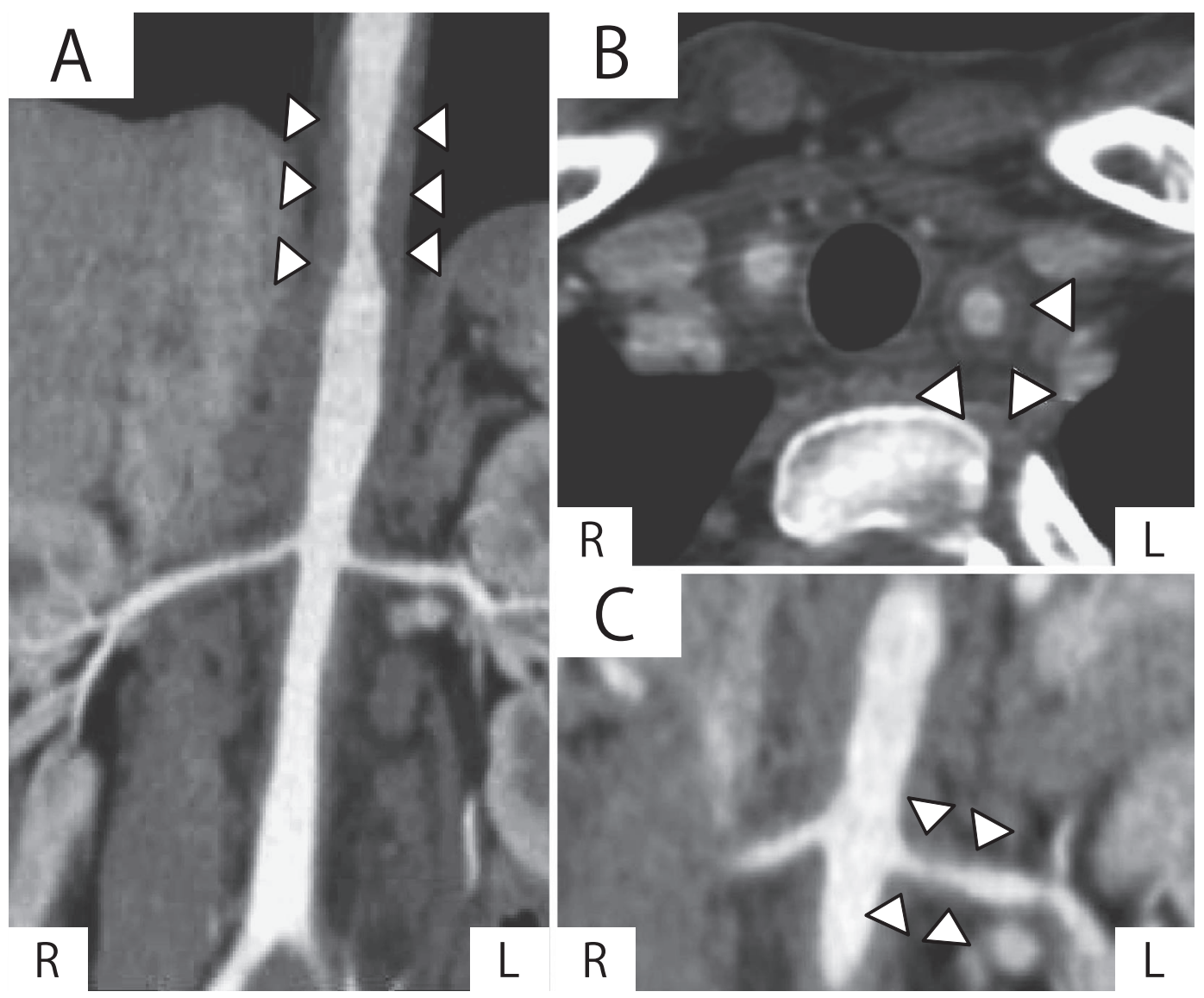

Figure 1. Contrast-enhanced CT at diagnosis of Takayasu's arteritis showed stenotic lesions of the thoracic and abdominal aorta (A) and its main branches, the common carotid artery (B), and the bilateral renal arteries (C). Arrows indicate radiological stenotic lesions. A: coronal sequence of aorta. B: axial sequence of right common carotid artery. C: coronal sequence of renal arteries. L: left, $R$ : right

puted tomography (CT) revealed stenosis of the bilateral renal arteries and thickening of the abdominal artery walls (Fig. 1). When she presented at our outpatient clinic 1 month before the current headache event, the active inflammation of TA was suppressed and her medical condition was stable under treatment with prednisolone at a dose of 30 $\mathrm{mg} /$ day.

On arrival at the emergency department, a physical examination showed normal temperature, and hypertension with a bilateral difference in blood pressure (right: 181/78 $\mathrm{mmHg}$, left 152/64 $\mathrm{mmHg}$ ). She was alert, and focal neurologic signs were absent. The results of laboratory examinations, including inflammatory reaction, electrolytes and renal function, were normal. An initial cerebrospinal fluid (CSF) examination was also normal. CT of her head showed no parenchymal abnormalities, such as subarachnoid hemorrhage or cerebral hemorrhage.

Her hypertension had diminished the following day, whereas her headache remained. No further seizures developed after admission. Brain magnetic resonance (MR) imaging using fluid-attenuated inversion recovery (FLAIR) showed diffuse hyperintense bilateral cortical and subcortical regions (Fig. 2A, B). MR angiography (MRA) also revealed proximal narrowing of the right posterior cerebral artery (Fig. 2C). We considered cerebral artery vasoconstriction to be related to her acute hypertension, although angiitis of the central nervous system could not be completely ruled out. We observed her under continuous treatment with $30 \mathrm{mg}$ prednisolone, based on an absence of neurologic symptoms, no fever, and normal CSF findings. On day 10 after her hospital admission, she had a recurrence of severe thunderclap headache with increased blood pressure, while straining during a bowel movement (right: 220/124 mmHg). On day 13, repeated MRA revealed an increase in the number of narrowed regions in the bilateral middle and posterior cerebral arteries, although the overall high intensity on FLAIR had disappeared (Fig. 2D, E, F). An ultrasound study of the internal carotid artery revealed no thickening of the vessel wall, although the wall of the common carotid artery was thickened on contrast-enhanced CT at TA diagnosis. Transcranial Doppler showed markedly increased blood flow velocity with turbulence in the right middle cerebral artery (mean blood flow velocity: right $134 \mathrm{~cm} / \mathrm{s}$, left $82 \mathrm{~cm} / \mathrm{s}$ ). Heparin was intravenously administered, and her systolic blood pressure was controlled below $150 \mathrm{mmHg}$ with nimodipine. Within 2 weeks of starting treatment, her head- 

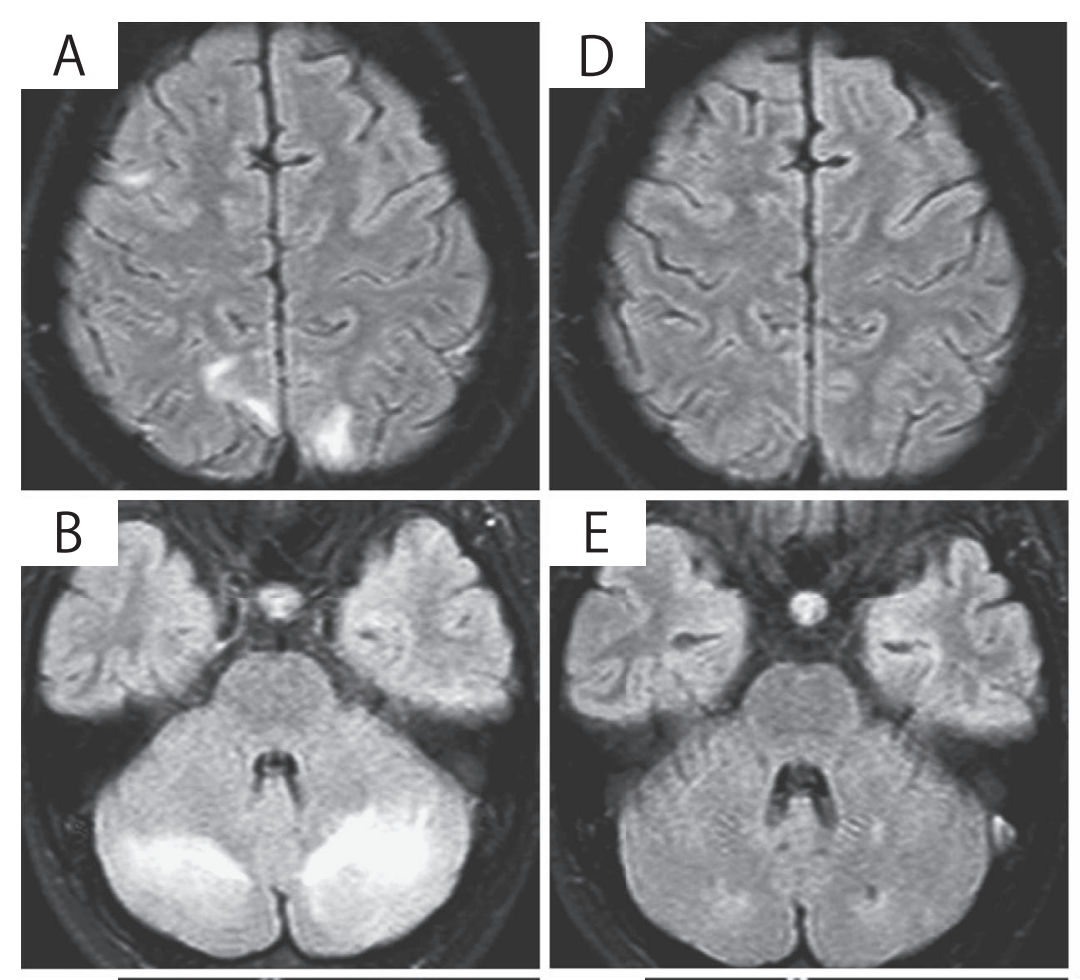

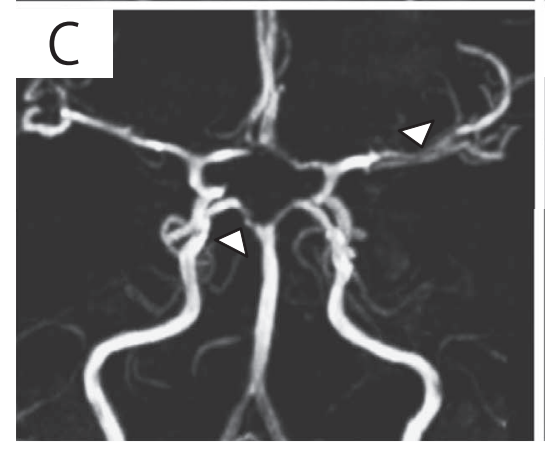

Day 3

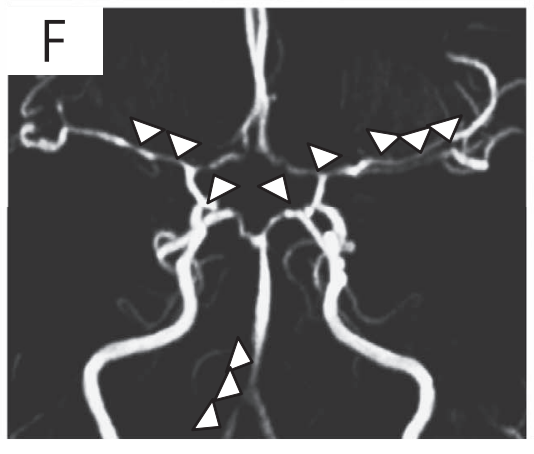

Day 13

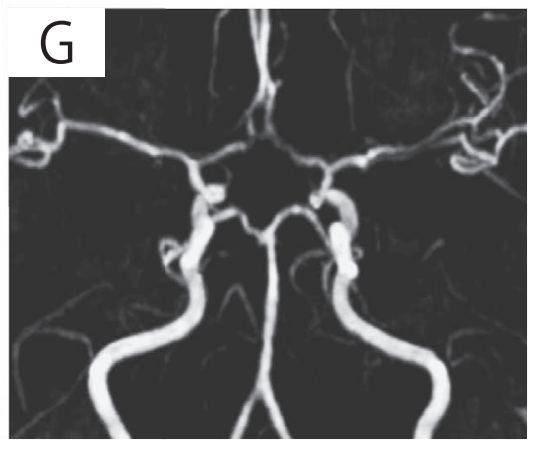

Day 29

Figure 2. Initial fluid-attenuated inversion recovery magnetic resonance (MR) image on day 3 showed hyperintense signals in the cortical and subcortical white matter, involving the bilateral cerebellar hemispheres, bilateral-occipital lobes and right frontal lobe (A, B). MR angiography (MRA) showed proximal narrowing of the right posterior cerebral artery (arrow) (C). Repeated MRA on day 13 revealed progressive luminal narrowing in multiple regions (arrows), although the overall parenchymal abnormality had disappeared (D, E, F). The cerebral narrowing in these areas was improved on follow-up MRA on day 29 (G).

ache gradually improved. MRA was significantly improved by day 29 after admission (Fig. 2G), and the blood flow velocity fell to normal (mean blood flow velocity: right 102 $\mathrm{cm} / \mathrm{s}$, left $81 \mathrm{~cm} / \mathrm{s}$ ). MRA revealed normal findings for all intracranial vessels 3 months after onset.

\section{Discussion}

The present case can be summarized as follows: a teenage girl receiving prednisolone therapy presented with thunderclap headache, decreased consciousness, convulsion and hypertension. Head MR imaging showed abnormal signals in the brain parenchyma and narrowing of the cerebral arteries, suggesting radiologic posterior reversible encephalopathy syndrome (PRES) and RCVS. The narrowing of the cerebral arteries progressed for 1 month, after which her symptoms rapidly improved and the abnormal signal detected by MR imaging diminished. To our knowledge, this is the first case of RCVS presenting in a patient with TA.

The criteria for RCVS were initially proposed by Calabrese et al (1) in 2007, as follows: i) transfemoral angiography or indirect CT angiography or MRA documenting multifocal segmental cerebral artery vasoconstriction; ii) no evidence of aneurysmal subarachnoid hemorrhage; iii) normal or near-normal CSF analysis; iv) severe, acute headache, with or without additional neurologic signs or symptoms; and v) reversibility of angiographic abnormalities within 12 weeks after onset. The symptoms and clinical data 
in the current patient matched all five of these original criteria. However, these criteria were unable to completely exclude the narrowing of intracranial cerebral arteries caused by primary angiitis-related TA in this case.

TA is an idiopathic granulomatous vasculitis that affects the aorta and its main branches. Approximately 10-15\% of patients with TA have ischemic stroke or transient ischemic attack. These strokes have mainly been attributed to stenotic extracranial vessels, caused by granulomatous vasculitis, whereas two cases of TA were reported as strokes involving the intracranial arteries $(4,6)$. The present case presented with stable symptomatic TA, with normal serological data and CSF findings. Moreover, an ultrasound study of the internal carotid artery revealed no thickening of the vessel wall. These observations suggested that the TA activity in this case was adequately suppressed and clinically controlled. The radiologically-detected intracranial cerebral artery narrowing had disappeared within several weeks, possibly suggesting that it was not related to the inflammatory reaction. This case was complicated by acute-onset headache and seizure, with increased blood pressure; patients with RCVS often complain of neurologic symptoms and a rapid rise in blood pressure. Thus, RCVS, rather than angiitis related to TA, might have been involved in the narrowing of the intracranial cerebral arteries in the current case.

PRES is a new clinicoradiologic entity characterized by headache, confusion, visual disturbance, seizures and transient reversible changes on MR imaging in the cortical and subcortical white matter of the parieto-occipital lobes $(7,8)$. PRES has previously been described in several conditions, including hypertensive encephalopathy, pre-eclampsia, eclampsia, infections, electrolyte imbalance, hypercalcemia and use of several therapeutic agents (7). Vasogenic edema based on endothelial dysfunction, occurring as a result of acutely elevated blood pressure exceeding the autoregulatory capacity of the brain vasculature, may be an essential factor in the development of PRES. In the current case, the neuroradiological finding and clinical course were consistent with the disease concept of PRES. The development of endothelial dysfunction in patients with TA might account for the ischemic signals and high intensity on FLAIR images, although the involvement of anti-endothelial antibody in TA remains unknown.
RCVS and PRES may have similar underlying mechanisms and may represent overlapping clinical entities, although they are cliniconeuroradiologically defined as distinct entities, for descriptive purposes. The rapid increase in blood pressure in the present case may, at least in part, have also played a crucial role in triggering vasoconstriction. Interestingly, patients with TA may present a clinical course similar to RCVS. An identical pathogenesis, inducing intracranial artery vasoconstriction, may also present different radiologic findings, with different regions of vasoconstriction and degrees of ischemic signals in the brain parenchyma, as in the two headache attacks in the present case.

In conclusion, we herein report a patient with TA complicated by RCVS. When a patient with TA presents with thunderclap headache with vascular abnormalities in intracranial vessels, the transient reactive vascular abnormality, RCVS, should be considered as a more likely differential diagnosis than organic stenotic granulomatous vasculitis.

The authors state that they have no Conflict of Interest (COI).

\section{References}

1. Calabrese LH, Dodick DW, Schwedt TJ, Singhal AB. Narrative review: reversible cerebral vasoconstriction syndromes. Ann Intern Med 146: 34-44, 2007.

2. Ducros A, Bousser MG. Reversible cerebral vasoconstriction syndrome. Pract Neurol 9: 256-267, 2009.

3. Arend WP, Michel BA, Bloch DA, et al. The American College of Rheumatology 1990 criteria for the classification of Takayasu arteritis. Arthritis Rheum 33: 1129-1134, 1990.

4. Kerr GS, Hallahan CW, Giordano J, et al. Takayasu arteritis. Ann Intern Med 120: 919-929, 1994.

5. Klos K, Flemming KD, Petty GW, Luthra HS. Takayasu's arteritis with arteriographic evidence of intracranial vessel involvement. Neurology 60: 1550-1551, 2003.

6. Takano K, Sadoshima S, Ibayashi S, Ichiya Y, Fujishima M. Altered cerebral hemodynamics and metabolism in Takayasu's arteritis with neurological deficits. Stroke 24: 1501-1506, 1993.

7. Fujita M, Komatsu K, Hatachi S, Yagita M. Reversible posterior leukoencephalopathy syndrome in a patient with Takayasu arteritis. Mod Rheumatol 18: 623-629, 2008.

8. Hinchey J, Chaves C, Appignani B, et al. A reversible posterior leukoencephalopathy syndrome. N Engl J Med 334: 494-500, 1996.

(C) 2011 The Japanese Society of Internal Medicine http://www.naika.or.jp/imindex.html 